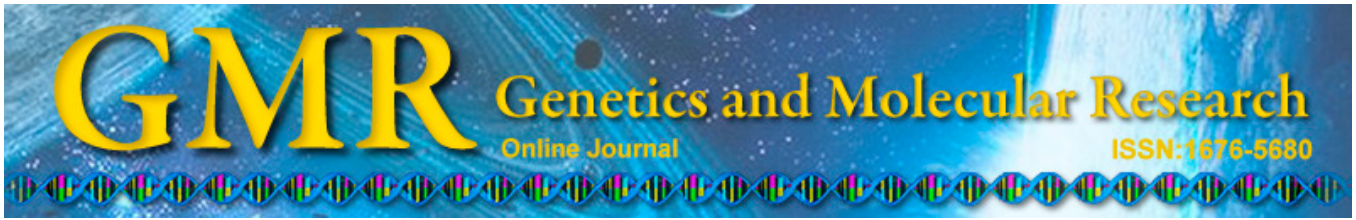

\title{
Correlation between $D Q B 1$ genetic polymorphism and genetic susceptibility in patients diagnosed with irritable bowel syndrome with diarrhea
}

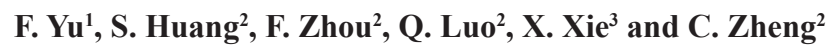 \\ ${ }^{1}$ Traditional Chinese Medicine Institute, Guangdong Medical College, \\ GuangDong, China \\ ${ }^{2}$ Guangdong Provincial Hospital, University City Branch, GuangDong, China \\ ${ }^{3}$ Guangdong HTCM Hospital, GuangDong, China \\ Corresponding authors: F. Yu / X. Zheng \\ E-mail: yujoyce022@163.com / xuebaozheng@sina.com.cn
}

Genet. Mol. Res. 13 (4): 10285-10293 (2014)

Received January 22, 2014

Accepted June 26, 2014

Published December 4, 2014

DOI http://dx.doi.org/10.4238/2014.December.4.23

\begin{abstract}
We examined patients of Han nationality diagnosed with irritable bowel syndrome with diarrhea (IBS-D) in Guangdong, China, to analyze the correlation between $D Q B 1$ allele polymorphisms and the genetic susceptibility to IBS-D. A total of 120 IBS-D patients of Han nationality in Guangdong, China, and 60 healthy control volunteers were included. $D Q B 1$ allele polymorphisms were investigated by polymerase chain reaction. Subjects' serum interleukin (IL)-10 level, colonic permeability, and tight junction marker zonula occludens 1 (ZO1) mRNA level were also investigated. Our data showed that the $D Q B 1^{*} 02$ allele frequency was significantly higher in IBS-D patients, while the $D Q B 1^{*} 0603$ frequency was lower than in healthy volunteers. The $D Q B 1 * 03, D Q B 1 * 04, D Q B 1 * 05, D Q B 1 * 0601, D Q B 1 * 0602$, and
\end{abstract}


$D Q B 1 * 0604$ alleles did not show significant differences between IBS-D patients and healthy controls. Furthermore, patients with $D Q B 1 * 03-$ positive and $D Q B 1 * 0603$-negative alleles showed more severe colonic permeability and lower serum IL-10 level and ZO1 level compared to healthy controls or even IBS-D patients with other genotypes. The present study indicated the $D Q B 1 * 02$ or $D Q B 1 * 0603$ alleles are related to IBS-D occurrence in Guangdong, China, and the mechanism of the disease may be related to reduced serum IL-10 levels.

Key words: Human leucocyte antigen- $D Q B 1$ gene; Interleukin-10; Irritable bowel syndrome with diarrhea

\section{INTRODUCTION}

Irritable bowel syndrome (IBS) is a chronic, recurrent intestinal problem, characterized by bloating, abdominal pain, and changes in bowel habits (Chang et al., 2010). The prevalence of IBS is approximately $10 \%$ in the Western population and $7.5 \%$ in the Chinese population (Park et al., 2010). The Rome III criteria classify the IBS phenotype into 4 groups: IBS with diarrhea (IBS-D), IBS with constipation (IBS-C), mixed IBS (IBS-M), and un-subtyped IBS (IBS-U). Although the pathophysiology of IBS is not fully understood, several mechanisms have been proposed, such as altered intestinal motility, visceral sensitivity, alterations in the two-way communication between the brain and gut, stress, and psychological factors (Arrieta et al., 2006; Aerssens et al., 2008; Sun et al., 2012). Recent studies have suggested immune system dysfunction as an important fundamental factor underlying the risk factors of IBS occurrence (Bertiaux-Vandaële et al., 2011). In addition, celiac disease, which has been defined as an immune dysfunctional disease and is associated with specific human leukocyte antigen (HLA) DQ genotypes, was suggested to be screened in patients with IBS-D (Locke et al., 2004), indicating that there are common features between these 2 diseases.

HLA-DQ (DQ) is a cell surface receptor type protein found on antigen-presenting cells and is an $\alpha \beta$ heterodimer of the major histocompatibility complex class II type (Chan et al., 2001). The $\alpha$ and $\beta$ chains are encoded by $H L A-D Q A 1$ and $H L A-D Q B 1$, respectively. Two $\alpha$-chain and $2 \beta$-chain variants, and thus 4 DQ isoforms, are frequently produced. As an antigen-presenting molecule, different $\mathrm{DQ}$ isoforms can bind to and present different antigens to T-cells or B-cells, thus stimulating the production of cytokines and antibodies. DQ is also involved in recognizing common self-antigens and presenting these antigens to the immune system in order to develop autoimmune tolerance.

Since their discovery in the 1970s, $H L A$ genes have been linked to more than 100 diseases, most of which are autoimmune diseases (Megiorni and Pezzuti, 2012). However, no linked $H L A-D Q B$ gene has been identified among IBS-D patients. In the present study, we investigated 120 patients diagnosed with IBS-D from the Han nationality in Guangdong, China and found that the $D Q B 1 * 02$ allele frequency was significantly higher and the $D Q B 1 * 0603$ frequency was lower in IBS-D patients compared to controls; furthermore, we found lower serum tumor necrosis factor- $\alpha$ and interleukin (IL)-10 levels in IBS-D patients. Our findings establish a new link between HLA-DQB and IBS-D and suggest that the HLS-DQB polymorphism is related to cytokine production. 


\section{MATERIAL AND METHODS}

\section{Subjects}

A total of 120 IBS-D subjects fulfilled the Rome III IBS diagnosis standard. Exclusion criteria included: 1) patients under 18 or over 65 years old; 2) pregnant and lactating women; 3) patients with intestinal organ diseases; 4) patients with tumor or severe diseases of the cardiovascular system, liver, kidney, or hematopoietic system; 5) patients who had undergone digestive tract surgery; 6) patients with severe primary disease or mental disease; 7) patients taking drugs to reduce gastrointestinal function or visceral sensitivity; and 8) patients taking drugs not as prescribed, resulting in efficacy assessment failure or inadequate information for efficacy and safety assessment. The patients included had no family medical history and the clinical information of the patients was registered. The 60 subjects in the control group were healthy volunteers without autoimmune diseases or a history of family genetic disease. All subjects were of the Han nationality in Guangdong, China. The study protocol was approved by the Research Committee of the Faculty of Medicine of the Southern Medical University.

\section{Genotyping}

\section{Reagents}

The dNTPs, Taq polymerase, Biowest Agarose, and genome DNA extraction reagent kit were purchased from Takara Biotechnology (Dalian Co., Ltd., Shiga, Japan). Isopropyl alcohol and absolute ethanol were purchased from Guangzhou Chemical Reagent Factory (Guangzhou, China). Human IL-10 (D1000B) enzyme-linked immunosorbent assay (ELISA) kits were purchased from R\&D Systems (Minneapolis, MN, USA).

\section{Template DNA preparation and HLA-DQB1 polymerase chain reaction (PCR) genotyping}

A venous blood sample was collected from subjects that had signed informed consent statements to participate in the study. Genomic DNA was isolated from whole blood drawn in ethylenediaminetetraacetic acid using a mixture of $5 \mathrm{~mL}$ buffer G2 (QIAamp DNA Blood Midi Kit; Qiagen; Hilden, Germany) and $95 \mathrm{~mL} 20 \mathrm{mg} / \mathrm{mL}$ proteinase K. After incubation at $50^{\circ} \mathrm{C}$ for $1 \mathrm{~h}$, the DNA was precipitated using ethanol, collected using a glass rod, and transferred into distilled water. The DNA concentration and quality were evaluated using a NanoDrop ND-1000 spectrometer (Thermo Fisher Scientific Inc., Waltham, MA, USA). Sequencespecific oligonucleotide probes were used with Luminex Xmap technology in order to identify the $H L A-D Q B$ allele groups of the individuals studied, as previously described (Gonsalkorale et al., 2003). This study was performed in a blinded manner.

\section{Measurement of colonic permeability}

For this test, $1000 \mathrm{mg}$ lactulose and $200 \mathrm{mg}$ mannitol (catalog No. L7877 and M8429, respectively; Sigma-Aldrich, St. Louis, MO, USA) were used to determine urine sugar excretion at different time points as markers of small bowel permeability after oral ingestion of the 
sugars in aqueous solution. First, $500 \mathrm{~mL}$ water was administered $30 \mathrm{~min}$ after sugar administration to aid in the collection of urine. Urine was collected every $30 \mathrm{~min}$ for the first $2 \mathrm{~h}$ (when the participant was able to provide a specimen, and cumulated for the entire $2 \mathrm{~h}$ ), and every $2 \mathrm{~h}$ for the next $6 \mathrm{~h}$. The total volume of each collection was measured, and an aliquot from each collection was obtained to estimate the total content of each sugar over the different time intervals. The urine aliquot was stored at $-20^{\circ} \mathrm{C}$ until it was thawed for analysis.

We estimated the cumulative and ratio excretion of the 2 sugars at $0-2$ and $8-24 \mathrm{~h}$ for colonic mucosal permeability, respectively, based on recent validation studies. Urinary saccharide concentrations were measured by high-performance liquid chromatography-tandem mass spectrometry. Cumulative excretion was calculated as the concentration of sugar $(\mu \mathrm{g} / \mathrm{mL}) \mathrm{x}$ total urine volume $(\mathrm{mL})$. The lactulose-to-mannitol ratio was calculated as $0.2 \mathrm{x}$ [(cumulative excretion of lactulose) / (cumulative excretion of mannitol)].

\section{Quantitation of tight junction (TJ) proteins by real-time PCR}

We used real-time PCR to determine the TJ level in rectosigmoid small bowel biopsy samples from IBS-D patients and 16 healthy subjects collected in a prior study. Rectosigmoid colon biopsy samples were submerged in TRIZOL (Invitrogen, Carlsbad, CA, USA) and stored at $-80^{\circ} \mathrm{C}$. After mRNA extraction, reverse transcription was conducted with 200 U M-MLV RT reagent (Promega, Madison, WI, USA) at $42^{\circ} \mathrm{C}$ for 60 min according to the manufacturer recommendations. The mRNA levels of zonula occludens $1(Z O-1)$ and actin were determined by SYBR green real-time PCR using the LightCycler Real-time PCR System (Roche Molecular Biochemicals, Mannheim, Germany).

The primer sequences were as follows: ZO-1 sense: 5'- GCA GCC ACA ACC AAT TCA TAG-3'; anti-sense: 5'-GCA GAC GAT GTT CAT AGT TTC G-3'; actin sense 5'-ACG GCC AGG TCA TCA CTA TTG-3' and anti-sense: 5'-ATG CCA CAG GAT TCC ATACCC-3'.

\section{ELISA}

The concentrations of IL-10 in colon tissue sample were determined using ELISA kits (R\&D Systems) according to the manufacturer instructions

\section{Statistical analysis}

Individuals normally have 2 of the $D Q B 1$ alleles, which can be either heterozygous or homozygous. The $D Q B 1$ allele frequencies of IBS-D and controls were calculated as the percentage of a particular allele relative to total alleles. Statistical analysis was performed using SPSS 13.0 for windows (SPSS, Inc., Chicago, IL, USA).

\section{RESULTS}

\section{Clinical features}

Among the 120 IBS-D patients, 52 were male (43.33\%) and 68 were female (56.67\%) and ranged in age from 18-56 years (average of 37.4 years). Symptoms lasted from 1.5-15 
years (average of 7.5 years). Patients and control groups had similar age range, body mass index, and gender distribution ( $\mathrm{P}>0.05$ for all). For the intestinal symptoms evaluated using a questionnaire, abdominal pain and discomfort were more frequent in subjects with IBS-D compared with controls, as expected. Similarly, the presence of loose stools, straining, the sensation of incomplete evacuation, mucus in the stools, and the sensation of abdominal fullness, inflammation, or swelling were more frequent in subjects with IBS-D (Table 1).

Table 1. General characteristics of the study groups.

\begin{tabular}{|c|c|c|c|}
\hline & Control $(\mathrm{N}=60)$ & IBS-D $(\mathrm{N}=120)$ & $P$ value \\
\hline Gender: female [N, (\%)] & $56(93.33)$ & $68(56.7)$ & 0.596 \\
\hline Age (years, mean) & 35.2 & 37.4 & 0.223 \\
\hline BMI (mean) & 24.4 & 25.7 & 0.742 \\
\hline \multicolumn{4}{|l|}{ BMI (categories \%) } \\
\hline Underweight & 3.3 & 1.6 & \\
\hline Normal & 45 & 61.7 & \\
\hline Overweight & 35 & 30 & \\
\hline Obese & 16.7 & 6.7 & \\
\hline \multicolumn{4}{|l|}{ Clinical characteristics according to the questionnaire (\%) } \\
\hline Abdominal pain or discomfort in the last 3 months & 28.3 & 100 & 0.001 \\
\hline Pain or discomfort improves or disappears after a bowel movement & 12.9 & 53.6 & 0.001 \\
\hline $\begin{array}{l}\text { When the abdominal pain or discomfort begins, } \\
\text { there are changes in the frequency of bowel movements }\end{array}$ & 0 & 78.3 & 0.001 \\
\hline $\begin{array}{l}\text { When the abdominal pain or discomfort begins, there } \\
\text { are changes in stool consistency }\end{array}$ & 2.3 & 96.4 & 0.001 \\
\hline Fewer than 3 bowel movements per week & 12.2 & 36.4 & 0.533 \\
\hline More than 3 bowel movements per day & 25.2 & 77.9 & 0.044 \\
\hline Hard or lumpy stools & 18.4 & 0 & 0.001 \\
\hline Loose, mushy, or watery stools & 22.5 & 100 & 0.015 \\
\hline Straining during a bowel movement & 1.7 & 0 & 0.001 \\
\hline Defecation urgency & 36.4 & 79.3 & 0.024 \\
\hline Sensation of incomplete evacuation & 11.5 & 28.4 & 0.013 \\
\hline Mucus in stools & 42.1 & 87.4 & 0.001 \\
\hline Sensation of abdominal fullness, inflammation, or swelling & 29.4 & 91.2 & 0.023 \\
\hline
\end{tabular}

Table 2. Frequency of $D Q B 1$ alleles in patients with IBS-D and controls.

\begin{tabular}{lccc}
\hline Alleles & \multicolumn{2}{c}{ Allele frequency } & Allele frequency analysis \\
\cline { 2 - 4 } & Control (\%) & IBS-D & P value \\
\hline$D Q 2(* 0201-* 0203)$ & $18(15.0)$ & $62(25.8)$ & 0.04 \\
$D Q 4(0401-0402)$ & $6(5.0)$ & $12(5.0)$ & 0.42 \\
$D Q 5(0501-0504)$ & $20(16.7)$ & $36(15.0)$ & 0.32 \\
$D Q 6-0601$ & $5(4.1)$ & $11(4.6)$ & 0.44 \\
$D Q 6-0602$ & $5(4.1)$ & $14(5.8)$ & 0.08 \\
$D Q 6-0603$ & $14(11.7)$ & $12(5.0)$ & 0.02 \\
$D Q 6-0604$ & $3(2.5)$ & $6(2.5)$ & 0.58 \\
$D Q 7(0301,0304)$ & $40(33.3)$ & $67(27.9)$ & 0.08 \\
$D Q 8(0302,0305)$ & $3(2.5)$ & $8(3.3)$ & 0.27 \\
$D Q 9(0303)$ & $6(5.0)$ & $12(5.0)$ & 0.88 \\
$\mathrm{~N}$ & 120 & 240 & \\
\hline
\end{tabular}

Numbers in parentheses are the percent of cases positive for the allele, and the number to the left of those are the number of cases positive for that allele.

\section{$H L A-D Q$ genotyping}

Table 2 shows the $H L A-D Q B 1$ allele frequencies in the IBS-D and healthy control groups. Compared with the healthy control group, IBS-D patients showed a higher frequency 
of the $D Q B 1^{*} 02$ allele and lower frequency of $D Q B 1 * 0603$. The frequencies of $D Q B 1 * 03$, $D Q B 1 * 04, D Q B 1 * 05, D Q B 1 * 0601, D Q B 1 * 0602$, and $D Q B 1 * 0604$ showed no significant differences.

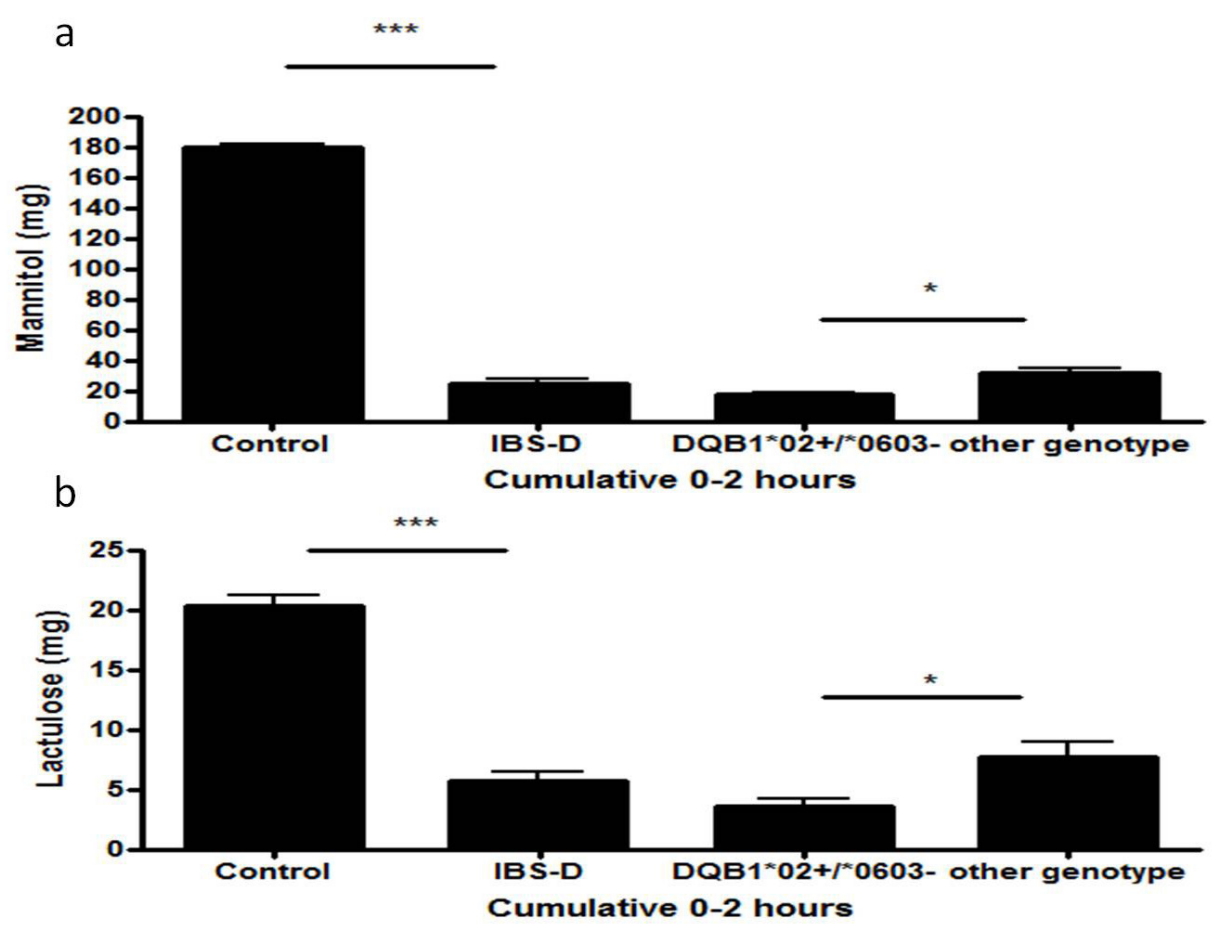

Figure 1. Comparisons of irritable bowel syndrome with diarrhea (IBS-D) and healthy control of small bowel permeability $[0-2 \mathrm{~h}$ cumulative excretion of mannitol and lactulose (both $\mathrm{P}<0.001$ )]. Values are reported as means $\pm \mathrm{SE}$.

Table 3. Demographics and permeability in control, $H L A-D Q B 1 * 02(+) / * 0603(-)$ IBS-D patients, and other IBS-D patients.

\begin{tabular}{lccc}
\hline & Control & IBS-D: $H L A-D Q B 1 * 02(+) / * 0603(-)$ & IBS-D: Other genotype \\
\hline $\mathrm{N}$ & 60 & 28 & 92 \\
$\mathrm{Age}$ & 35.2 & 34.7 & 38.1 \\
BMI & 24.4 & 21.5 & 26.5 \\
Cumulative urine mannitol (mg) & 179.4 & 18 & 31.56 \\
Cumulative urine lactulose (mg) & 20.35 & 3.55 & 7.76 \\
\hline
\end{tabular}

\section{IBS-D patients showed increased small bowel permeability}

IBS-D patients showed higher small bowel permeability compared with healthy controls. Interestingly, when the data were organized by genotype subgroups, it was found that IBS-D patients with the $D Q B 1^{*} 02(+) / D Q B 1 * 0603(-)$ alleles showed even more severe bowel permeability compared with healthy controls or IBS-D patients with other genotypes (Figure 1, Table 3). These observations were based on urine mannitol and lactulose excretion from $0-2 \mathrm{~h}$ (both $\mathrm{P}<0.001$ ). 
a

zonula occludens-1 (ZO-1) mRNA level

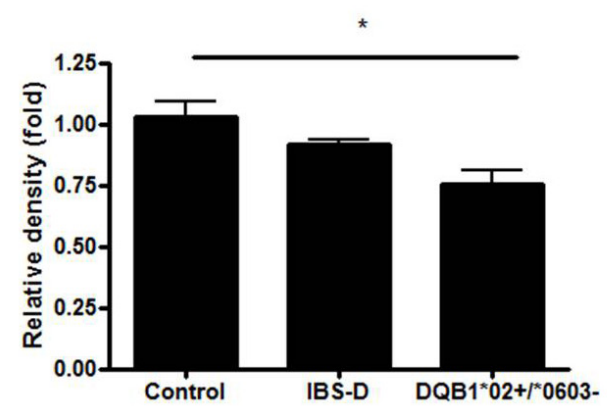

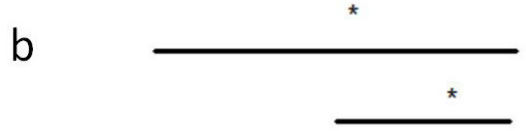

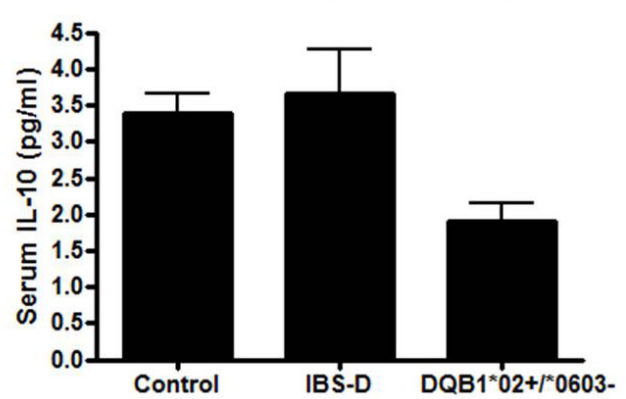

Figure 2. a. $Z O 1$ mRNA level in healthy controls, IBS-D patients, and some genotypes of IBS-D patients; $\mathbf{b}$. serum IL-10 level in healthy controls, IBS-D patients, and certain genotype IBS-D patients. ${ }^{*} \mathrm{P}<0.05$.

\section{IBS-D patients with $D Q B 1 * 02(+) / D Q B 1 * 0603(-)$ alleles showed lower $Z O-1$ mRNA and serum IL-10 levels}

We further analyzed ZO-1 mRNA and serum IL-10 levels. When considered as a whole group, IBS-D patients showed no significant difference in ZO-1 mRNA and serum IL-10 levels compared with healthy controls, while IBS-D patients with $D Q B 1 * 02(+) / D Q B 1 * 0603(-)$ alleles showed significantly reduced levels of these markers (Figure $2 a, b)$.

\section{DISCUSSION}

In the present study, we evaluated the influence of $H L A-D Q B$ genotypes on 120 different IBS-D patients of the Han nationality, and then investigated small bowel barrier function, the level of the tight junction marker ZO-1, and serum IL-10 level. Our data showed that in IBS-D patients, the frequency of the $D Q B 1^{*} 02$ allele was significantly higher than that in healthy volunteers, while the $D Q B 1^{*} 0603$ frequency was lower. All other $D Q B$ genotypes measured showed no significant difference. Furthermore, we showed that IBS-D patients' small bowel mucosal function was impaired, which is consistent with the results of a previous report. In addition, we also found that ZO-1, a tight junction marker that indicates mucosal function, was reduced in IBS-D patients compared with healthy controls, but was further decreased in $D Q B 1^{*} 02 / * 0603(+/-)$ patients. Finally, we found that the serum tumor necrosis factor- $\alpha$ level was increased, while the IL-10 level was decreased in $D Q B 1^{*} 02 / * 0603(+/-)$ patients compared to healthy controls and other IBS patients.

IBS is a common clinical entity of chronic bowel dysfunction and has a high incidence. Epidemiological studies indicate familial inheritance in IBS patients and a higher risk rate of IBS co-occurrence in monozygotic twins than in dizygotic twins (Neagoe et al., 2004), suggesting that genetic susceptibility is likely responsible for IBS incidence; thus, screening for IBS-related susceptible genes is important. Wahnschaffe et al. (2007) and Adler et al. (2006) investigated the correlation between diarrhea-predominant IBS and HLA-DQA1*0501/ $D Q B 1^{*} 0201$ and found a high rate of occurrence of $H L A-D Q 2$ in diarrhea-predominant IBS. 
The major histocompatibility complex, HLA, is a gene cluster closely related to immune response (Liu et al., 2009). HLA-DQB1 belongs to the HLA class II beta chain paralogs and plays a central role in the immune system by presenting peptides derived from exogenous proteins to immune cells. Variant transcripts are encoded by $H L A-D Q B 1$ genes and are the result of single-nucleotide polymorphisms (SNPs). When SNPs result in amino acids changes in functional regions, DQB isoforms are produced. In general, variant DQB isoforms can bind to different peptides and then present to T-cells, thus stimulating an immune response to variant exogenous peptides. In the present study, we found that in IBS-D patients, the frequency of the $D Q B 1^{*} 02$ genotype was significantly higher than that in healthy controls, while the $D Q B 1 * 0603$ frequency was significantly lower.

Although the roles of $H L A-D Q B$ status and potential immunogenetic mechanisms in symptom generation in IBS-D patients remain controversial, there is some evidence in favor of immunogenetic mechanisms, including the fact that $H L A-D Q 8$ knockout transgenic mice sensitized to gluten and gliadin exposure showed increased contraction of smooth muscle to electrical field stimulation and carbachol (Verdu et al., 2008). In addition, patients with IBS-D had accelerated colonic permeability compared with healthy controls (Vazquez-Roque et al., 2012). Another report showed that $45 \%$ of patients with the symptom phenotype of IBS-D have accelerated colonic bowel permeability (Camilleri et al., 2008). Here, we found that, in addition to the colon, IBS-D patients showed increased small bowel permeability. Although the underlying mechanisms remain unclear, we hypothesize that $D Q B$ polymorphisms may induce different immune responses related with IBS-D.

IBS patients were previously found to have a lower frequency of the high producer $I L$ 10 genotype compared with controls in Turkish patients (Gonsalkorale et al., 2003), and other studies have revealed that the presence of low serum levels of IL-10 is an independent predictive factor of IBS (Schmulson et al., 2013). In addition, women with IBS-D presented with lower IL-10 levels compared to those with IBS-C and IBS-M (Schmulson et al., 2012). In the present study, we found that when the entire population was considered, the IL-10 level did not differ significantly between ISB-D patients and healthy controls, but a $D Q B 1^{*} 02 / * 0603(+/-)$ subgroup of ISB-D patients showed a decreased serum IL-10 level compared with healthy controls and other IBS-D patients. Our results indicate that $D Q B 1$ genotypes may be related to differences in IL-10 levels.

Small bowel permeability is primarily controlled by the small bowel epithelial barrier, and barrier dysfunction is related to increased small bowel permeability (Vanderpool et al., 2008). Previous reports found that in an $I L-10$ knockout mice model, the atypical junction complex was impaired and colonic permeability was increased (Nahidi et al., 2013), indicating that serum IL-10 levels may be closely related to colonic function through regulating TJs. Here, we found that in the $D Q B 1 * 02 / * 0603(+/-)$ subgroup of ISB-D patients, the TJ marker $Z O-1$ level in the small bowel was significantly reduced compared to that in healthy controls and other ISB-D patients. This indicates that the $D Q B 1$ genotype may affect small bowel function by disturbing the general immune response.

In summary, we suggest that the $D Q B 1 * 02$ and $D Q B 1 * 0603$ alleles are susceptibility genes in IBS patients of Han nationality in Guangdong, China, which may be directly related to lower serum IL-10 levels, and thus reduce ZO-1 levels and increase colonic permeability. Our data provide supporting evidence for studies examining $H L A-D Q B 1$ gene susceptibility in IBS patients. 


\section{REFERENCES}

Adler SN, Jacob H, Lijovetzky G, Mulder CJ, et al. (2006). Positive coeliac serology in irritable bowel syndrome patients with normal duodenal biopsies: video capsule endoscopy findings and HLA-DQ typing may affect clinical management. J. Gastrointestin. Liver Dis. 15: 221-225.

Aerssens J, Camilleri M, Talloen W, Thielemans L, et al. (2008). Alterations in mucosal immunity identified in the colon of patients with irritable bowel syndrome. Clin. Gastroenterol. Hepatol. 6: 194-205.

Arrieta MC, Bistritz L and Meddings JB (2006). Alterations in intestinal permeability. Gut 55: 1512-1520.

Bertiaux-Vandaële N, Youmba SB, Belmonte L, Lecleire S, et al. (2011). The expression and the cellular distribution of the tight junction proteins are altered in irritable bowel syndrome patients with differences according to the disease subtype. Am. J. Gastroenterol. 106: 2165-2173.

Camilleri M, McKinzie S, Busciglio I, Low PA, et al. (2008). Prospective study of motor, sensory, psychologic, and autonomic functions in patients with irritable bowel syndrome. Clin. Gastroenterol. Hepatol. 6: 772-781.

Chan PK, Cheung JL, Cheung TH, Lin CK, et al. (2001). HLA-DQB1 polymorphisms and risk for cervical cancer: A casecontrol study in a southern Chinese population. Gynecol. Oncol. 105: 736-741.

Chang FY, Lu CL and Chen TS (2010). The current prevalence of irritable bowel syndrome in Asia. J. Neurogastroenterol. Motil. 16: 389-400.

Gonsalkorale WM, Perrey C, Pravica V, Whorwell PJ, et al. (2003). Interleukin 10 genotypes in irritable bowel syndrome: Evidence for an inflammatory component? Gut 52: 91-93.

Liu Y, Ye Z, Luo H, Sun M, et al. (2009). Inhalative formaldehyde exposure enhances aggressive behavior and disturbs monoamines in frontal cortex synaptosome of male rats. Neurosci. Lett. 464: 113-116.

Locke GR 3rd, Murray JA, Zinsmeister AR, Melton LJ 3rd, et al. (2004). Celiac disease serology in IBS and dyspepsia: a population-based case-control study. Mayo Clin. Proc. 79: 476-482.

Megiorni F and Pizzuti A (2012). HLA-DQA1 and HLA-DQB1 in Celiac disease predisposition: practical implications of the HLA molecular typing. J. Biomed. Sci. 19: 88-93.

Nahidi L, Leach ST, Mitchell HM, Kaakoush NO, et al. (2013). Inflammatory bowel disease therapies and gut function in a colitis mouse model. Biomed. Res. Int. 2013; 909613.

Neagoe A, Molnar AM, Acalovschi M, Seicean A, et al. (2004). Risk factors for colorectal cancer: an epidemiologic descriptive study of a series of 333 patients. Rom. J. Gastroenterol. 13: 187-193.

Park DW, Lee OY, Shim SG, Jun DW, et al. (2010). The differences in prevalence and sociodemographic characteristics of irritable bowel syndrome according to Rome II and Rome III. J. Neurogastroenterol. Motil. 16: 186-193.

Schmulson M, Pulido-London D, Rodríguez O, Morales-Rochlin N, et al. (2012). Lower serum IL-10 is an independent predictor of IBS among volunteers in Mexico. Am. J. Gastroenterol. 107: 747-753.

Schmulson M, Pulido-London D, Rodríguez O, Morales-Rochlin N, et al. (2013). IL-10 and TNF-alpha polymorphisms in subjects with irritable bowel syndrome in Mexico. Rev. Esp. Enferm. Dig. 105: 392-399.

Sun M, Zhou T, Zhou L, Chen Q, et al. (2012). Formononetin protects neurons against hypoxia-induced cytotoxicity through upregulation of ADAM10 and sA $\beta P P \alpha$. J. Alzheimers Dis. 28: 795-808.

Vanderpool C, Yan F and Polk DB (2008). Mechanisms of probiotic action: implications for therapeutic applications in inflammatory bowel diseases. Inflamm. Bowel Dis. 14: 1585-1596.

Vazquez-Roque MI, Camilleri M, Smyrk T, Murray JA, et al. (2012). Association of HLA-DQ gene with bowel transit, barrier function, and inflammation in irritable bowel syndrome with diarrhea. Am. J. Physiol. Gastrointest. Liver Physiol. 303: G1262-G1269.

Verdu EF, Huang X, Natividad J, Lu J, et al. (2008). Gliadin-dependent neuromuscular and epithelial secretory responses in gluten-sensitive HLA-DQ8 transgenic mice. Am. J. Physiol. Gastrointest. Liver Physiol. 294: G217-G225.

Wahnschaffe U, Schulzke JD, Zeitz M and Ullrich R (2007). Predictors of clinical response to gluten-free diet in patients diagnosed with diarrhea-predominant irritable bowel syndrome. Clin. Gastroenterol. Hepatol. 5: 844-850. 